\title{
Dynamics of fast electron beams and bounded targets
}

\author{
N. Zabala ${ }^{1,2}$ and A. Rivacoba ${ }^{2,3}$ \\ ${ }^{1}$ Department of Electricity and Electronics, FCT-ZTF, \\ UPV/EHU, 48080 Bilbao, Spain. \\ ${ }^{2}$ Materials Physics Center CSIC-UPV/EHU and Donostia International Physics Center \\ DIPC, \\ Paseo Manuel Lardizabal 4, 20018 Donostia-San Sebastian, Spain. \\ ${ }^{3}$ Department of Materials Physics, Faculty of Chemistry, UPV/EHU, 20080 Donostia, \\ Spain. \\ We analyze the full relativistic force experienced by swift electrons moving close to planar \\ films for the experimental conditions commonly used in electron energy loss spectroscopy \\ in STEM. In metals the main effects derive from the dispersion of the surface plasmons, \\ which are clearly observed in the EEL spectra. In insulators we explore the role played \\ by the Cherenkov radiation $(\mathrm{CR})$ emitted in the energy gap window. The focus is placed \\ on the transverse force and different factors which may turn this force into repulsive, as \\ reported in recent experimental and theoretical works.
}




\section{Introduction}

The retarding force experienced by electron probes in inhomogeneous media has attracted great attention in the last decades due to its interest in electron energy loss spectroscopy (EELS) in scanning transmission electron microscopy (STEM). Nowadays this technique has become a very accurate tool to characterize nano-structures and map plasmons with high resolution. The transverse component of the force has received less attention, but in general it is assumed to be attractive and small. Nevertheless, several experiments and theoretical predictions have questioned the expected behaviour.

Early experiments $[1,2]$ detected strong deflections when a $100 \mathrm{keV}$ electron beam passed close to targets of $\mathrm{MgO}$ and $\mathrm{Au}$ of $100 \mathrm{~nm}$ side at a distance of around $1 \mathrm{~nm}$, which stimulated different calculations of the image force within classical dielectric theory $[3,4]$, considering infinite planar surfaces or spherical targets [5]. More recently, advances in electron microscopy have allowed to monitor the movement of nanometric gold particles under the action of a well focused electron beam, finding that the force experienced by the particle becomes repulsive at small impact parameters. These experiments have stimulated new relativistic calculations of the momentum transferred to nanoparticles (NPs), accounting for a precise description of the target [6-8], which reproduce the observed repulsive interaction, but the physics behind this effect is still unclear. In fact this repulsive force near small metallic particles at small impact parameter was also reported by García de Abajo [9] in a previous work. In a very recent paper Rebernik [10] predicted that the interaction between a planar surface and a charge packet moving parallel to it becomes repulsive above a critical relativistic energy.

In the present work the retardation effects on the force between $300 \mathrm{keV}$ electron beams and metallic $(\mathrm{Al}, \mathrm{Au})$ or insulator $(\mathrm{MgO})$ films are explored, with the focus on the sign of the transverse component, extending a recent study for a semi-infinite medium [11]. First we analyze the effects of retardation induced plasmon dispersion clearly observable in the EEL spectra. In the case of insulator targets it has been proved that most of the relativistic effects derive from Cherenkov radiation (CR). The behavior of the transverse force with impact parameter is finally studied, finding that it is always attractive for the common STEM setups, but different factors providing repulsive forces are discussed. 


\section{Theory}

The Lorentz force experienced by a fast electron moving at velocity $\mathbf{v}$ and impact parameter $b$, paralell to a planar film of thickness $a$ (see inset of figure 1(a)), characterized by a dielectric function $\epsilon(\omega)$, is calculated following the same method used for semi-infinite targets in a previous reference [11]. Both transverse and longitudinal components are written in terms of a single complex response function $\Sigma$, which depends on frequency $\omega$ and parallel component of the momentum $k_{y}$ :

$$
\begin{aligned}
& F_{x}=\frac{-2}{\pi v} \int_{0}^{\infty} d k_{y} \int_{0}^{\infty} d \omega \nu_{0} \operatorname{Re}\left[\Sigma\left(\mathrm{k}_{\mathrm{y}}, \omega\right)\right] \mathrm{e}^{-2 \nu_{0} \mathrm{~b}} \\
& F_{z}=\frac{-2}{\pi v} \int_{0}^{\infty} d k_{y} \int_{0}^{\infty} d \omega \frac{\omega}{v} \operatorname{Im}\left[\Sigma\left(\mathrm{k}_{\mathrm{y}}, \omega\right)\right] \mathrm{e}^{-2 \nu_{0} \mathrm{~b}}
\end{aligned}
$$

where

$\Sigma\left(k_{y}, \omega\right)=\frac{\epsilon-1}{\Delta_{0}}\left[\frac{2 \nu_{0}}{\Delta} \Lambda-\frac{\omega^{2}}{c^{2}} \frac{1-\beta^{2}}{\nu_{0}}\left(1-e^{-2 \nu a}\right)\right]$,

and

$$
\begin{aligned}
& \Delta_{0}=\left(\nu+\nu_{0}\right)^{2}-\left(\nu-\nu_{0}\right)^{2} e^{-2 \nu a} \\
& \Delta=\left(\nu+\nu_{0} \epsilon\right)^{2}-\left(\nu-\nu_{0} \epsilon\right)^{2} e^{-2 \nu a} \\
& \Lambda=\left(\nu+\nu_{0}\right)\left(\nu+\nu_{0} \epsilon\right)+\left(\nu-\nu_{0}\right)\left(\nu-\nu_{0} \epsilon\right) e^{-4 \nu a}-2\left(\nu^{2}+\nu_{0}^{2} \epsilon\right) e^{-2 \nu a},
\end{aligned}
$$

with $\nu=\sqrt{k_{y}^{2}+\left[1-\beta^{2} \epsilon(\omega)\right] \omega^{2} / v^{2}}, \nu_{0}=\sqrt{k_{y}^{2}+\omega^{2} / \gamma^{2} v^{2}}, \beta=v / c$ and $\gamma=\left(1-\beta^{2}\right)^{-1 / 2}$ the Lorentz factor. Notice that these general equations lead to the simpler expressions for the semi-infinite medium [11] as $a \rightarrow \infty$. From one side, for EELS applications in electron microscopy, it is helpful to consider the $\omega$ dependent integrand in the longitudinal force $[12]$,

$F_{z}=-\int_{0}^{\infty} \omega d \omega \frac{d P}{d z d \omega}$

which corresponds to the probability $P$ (per unit length) of losing energy $w$,

$$
\frac{d P}{d z d \omega}=\frac{2}{\pi v^{2}} \int_{0}^{\infty} d k_{y} e^{-2 \nu_{0} b} \operatorname{Im}\left[\Sigma\left(\mathrm{k}_{\mathrm{y}}, \omega\right)\right]
$$

On the other side, in order to describe the nature (attractive or repulsive) of the interaction, it is illustrative to analyze the transverse momentum transfer $p_{x}$ per unit length and energy,

$$
\frac{d p_{x}}{d z d \omega}=\frac{F_{x}}{v d \omega}=-\frac{2}{\pi v^{2}} \int_{0}^{\infty} d k_{y} \operatorname{Im}\left[\mathrm{k}_{\mathrm{x}} \Sigma\left(\mathrm{k}_{\mathrm{y}}, \omega\right)\right] \mathrm{e}^{-2 \nu_{0} \mathrm{~b}} .
$$


Notice also that $\nu_{0}$ may be interpreted as the modulus of $k_{x}$, the transverse wave vector component associated to the evanescent fields, $k_{x}=i \nu_{0}=\sqrt{\omega^{2} c^{-2}-k_{y}^{2}-\omega^{2} v^{-2}}$.

The previous formalism provides a description of the scattering of the probe in terms of excitations of momentum $\mathbf{k}=\left(k_{x}, k_{y}, \omega v^{-1}\right)$ and energy $\omega$, where $\Sigma\left(k_{y}, \omega\right)$ is a key function describing the probability (always per unit length) of exciting such a process. In [11] we described in detail the behavior of this function for metals and insulators in a semi-infinte medium. It is worth mentioning that its imaginary part is always positive in the whole $\omega-k_{y}$ space, while its real part changes its sign around the surface plasmon dispersion curve.

Finally, notice that the non-retarded limits are directly recovered from these expressions for $\beta \rightarrow 0$.

Following reference [11] we have also calculated the force experienced by the film, finding that, for any dielectric function, it is equal to the force on the probe. It is important to consider that the total momentum transfer to the film can contain some radiative contributions in the case of CR supporting media.

\section{Results}

First we analyze the energy loss probability, which is the magnitude commonly measured in EELS experiments, and the effects of retardation in Al slabs of different thicknesses, represented by a Drude dielectric function $\epsilon(\omega)=1-\omega_{p}^{2} /\left(\omega^{2}+i \omega \gamma\right)$, with $\omega_{p}=15.3$ $\mathrm{eV}$ and $\gamma=1 \mathrm{eV}$. The effects of retardation in metals derive mainly from the dispersion of the surface plasmon $\omega\left(k_{y}\right)$, as described by equations (1) and (2) and discussed in [11] for semi-infinite planar targets. In that case the surface plasmon is red-shifted as $k_{y} \rightarrow 0$. For films there are two surface plasmon modes [13], symmetric $\left(\omega_{-}\right)$and antisymmetric $\left(\omega_{+}\right)$, which in the non-retarded limit are given by $\omega_{ \pm}=\omega_{p}\left[\left(1 \pm e^{-q a}\right) / 2\right]^{1 / 2}$, where $q=\left(k_{y}^{2}+\omega^{2} / v^{2}\right)^{1 / 2}$ is the parallel momentum. In the retarded case the modes (given by the zeros of the denominator of $\Sigma$ function) exhibit a shift towards the surface plasmon frequency of the semi-infinite medium as $k_{y} \rightarrow 0$, as is clearly observed in figure 1(b).

This behavior is illustrated in the EEL spectra of figure 1(a) for three different values of the thickness $a=1,10$ and $a=100 \mathrm{~nm}$, corresponding to $300 \mathrm{keV}$ electrons at impact parameter $b=1 \mathrm{~nm}$. In the last case of the thickest film the spectra are pretty similar to 
the ones obtained for a semi-infinite medium at the same impact parameter. Moreover, retardation produces a shift and broadening of the surface plasmon peaks, which is much stronger for thicker films. For $a=1 \mathrm{~nm}$ the non-retarded approximation works pretty well, as observed in the figure.

Furthermore, as the probe trajectory is more distant from the surface, the effects of retardation become stronger. This is because the small momenta have more weight in the loss spectrum, as inferred from the exponential factor in the $k_{y}$ integral in equation (2), and it is just at small $k_{y}$ where dispersion is more relevant, as shown by the dispersion curves described above.

In insulators, which are transparent in an energy range, there is an additional mechanism entering into play within the relativistic formalism: the emission of Cherenkov radiation. In the case of $\mathrm{MgO}$ this happens for $\omega<6.6 \mathrm{eV}$, where the dielectric function (we use experimented data [14]) is a pure real magnitude with a maximum value of 7.3 at the gap edge, decreasing to 3.2 as $\omega \rightarrow 0$. Then, for electron velocities such that $\epsilon \beta^{2}>1$, the momentum $\nu$ becomes purely imaginary and the term $e^{-\nu x}$ describes propagating electromagnetic fields in the medium. For $300 \mathrm{keV}(\beta=0.78)$ electrons considered in the present study the CR condition is fulfilled in the whole energy window.

The CR propagating fields are emitted forward, in a cone given by $\cos \theta_{C}=\beta^{-1} \epsilon^{-1 / 2}$. In the case of films, in contrast with the previously studied semi-infinite medium [11], the radiation is reflected at the interfaces producing standing waves propagating as in a planar waveguide. The radiative modes may be derived from the poles of the $\Sigma$ function, in the region where $\epsilon$ is real. Given that the main contribution in the $k_{y}$ integral arises from small values of this variable, it is easy to prove that the allowed modes satisfy the following condition:

$\omega=\frac{v}{a \sqrt{\left.\epsilon(\omega) \beta^{2}-1\right)}}(n \pi-2 \phi)$,

where $n=1,2,3 \ldots$ is an integer and $\phi=\arctan \left\{1 / \epsilon\left[\left(\epsilon(\omega) \beta^{2}-1\right) /\left(1-\beta^{2}\right)\right]^{1 / 2}\right\}$ a phaseshift. Notice that, neglecting the phase, equation (8) states that the transverse component of the wave vector of the allowed radiation satisfies the quantization condition for the transverse momentum, $k_{x}=n \pi / a$. A similar behavior was found within a cylindrical model used to explain EEL spectra in porous alumina [15] some years ago. This 
phenomenon is visualized in figure 2, where the calculated EEL spectra corresponding to $\mathrm{MgO}$ films of three different thicknesses $(a=10 \mathrm{~nm}, 100 \mathrm{~nm}$ and $1 \mathrm{~mm})$ are compared. The electron impact parameter is $b=1 \mathrm{~nm}$, except for the black curve in figure 2(c) corresponding to a thick film and $b=10 \mathrm{~nm}$. The non-retarded probabilities are show in dashed line for comparison. Notice that for the thinest film of $10 \mathrm{~nm}$, there is no excitation below $6.6 \mathrm{eV}$. In fact equation (8) states a minimum thickness of about $29 \mathrm{~nm}$ for the first $n=1$ mode to be activated. As the thickness increases more modes are excited at smaller energies, until the whole CR window is covered, so that for very thick films the spectrum approaches the continuum calculated for the corresponding semi-infinite medium, as shown in figure $2(\mathrm{c})$ corresponding to $a=1 \mathrm{~mm}$. The $\mathrm{CR}$ effects really dominate the EEL spectrum for distant trajectories, as it is observed for the case of $b=10 \mathrm{~nm}$, in which the main losses occur in the $\mathrm{CR}$ window.

Now we turn our attention to the transverse component of the force experienced by the probe and the different factors which might turn this force from attractive into repulsive, as reported in recent works $[7,10]$. In figure 3 results are shown for both $\mathrm{MgO}$ and $\mathrm{Au}$ films, always for $300 \mathrm{keV}$ electrons. For other metals as Al similar results are obtained. The dashed line, as in the previous figures, corresponds to the non-retarded calculations. Moreover, we notice that the non-retarded approximation overestimates the force, which is always attractive, except for figure 3(b) corresponding to Au. We observe that at impact parameters below $1 \mathrm{~nm}$, the force becomes repulsive when the experimental dielectric function extracted from [16] is used to describe the film. This result is an unphysical consequence of the non-causal character of the dielectric function used for this calculation, as found for semi-infinite media [11]. Causality turns out to be a key issue in this calculation, because the total force is obtained as an integral in the whole $\omega$ spectrum, $F_{x}=\int_{0}^{\infty} \mathcal{F}(\omega) d \omega$, where $\mathcal{F}(\omega)$ is an integral in $k_{y}$ involving the $\operatorname{Re}\left[\Sigma\left(\omega, \mathrm{k}_{\mathrm{y}}\right)\right]$ function. The real part of the $\Sigma$ function changes its sign around the surface resonances, so that when integration in $\omega$ is performed there are strong cancellations of contributions below and above its corresponding resonance energies [11]. The correct causal behavior of the dielectric function is critical at small $b$ because in this case the contributions from the high $\omega$ values to the total force is important, contrary to the case of distant trajectories. In order to check this point we have considered the parametrization for the Au dielectric 
function proposed by Werner et al. [17], which considers a set of nine Lorentzians and by construction fulfills the K-K relations. In this case we observe that the force is attractive also at very small impact parameters.

At large impact parameters only the low $k_{y}$ and $\omega$ contribute to the integrals and, as a consequence, all the contributions to the force are attractive (see inset of figure 3(a)), so that for $b \rightarrow \infty$ it is proved numerically that the quasi-static limit $F_{x} \rightarrow-\left(4 b^{2} \gamma\right)^{-1}$ is reached. Moreover, the non-retarded approximation overestimates the attractive force except for $b \rightarrow 0$ as the force becomes divergent in the local approximation of $\epsilon(\omega)$ considered. At $b \approx 10 \mathrm{~nm}$ we notice that both retarded and non-retarded expressions lead to the same value of the force. We observe again that retardation is more important for wider films, as it is evident from the comparison of $F_{x}$ for $a=1 \mathrm{~nm}$ and $a=10 \mathrm{~nm}$ in the case of $\mathrm{Au}$.

The importance of considering the contribution of the magnetic field in the Lorentz force calculated to compute $F_{x}$ also worths some attention. Even at lower probe energies than the one considered in the present work, we proved [11] that the only contribution of the electric field provides a repulsive force at small impact parameters, which is turned into attractive when the contribution of the magnetic field is added. Although the contribution of the magnetic field to the force is much smaller than that of the electric field, the cancellation effects in the $\omega$ spectrum explain this result [11].

Another point worth of attention is the width of the beam. In the present work we have considered a point electron beam, which is a good approach for the EELS experimental setup. Nevertheless, Rebernik [10] claimed that the lateral extension of the beam in the $y$ direction would turn the interaction repulsive for very swift electrons in a semi-infinite medium. This relativistic effect is confirmed for films within the present formalism and using a gaussian profile of the beam. The detailed expressions were given in a previous article for semi-infinite media [11]. Nevertheless, the beam widths required for such an effect are of the order of $100 \mathrm{~nm}$, a size which does not correspond to the beams currently used in electron microscopy experiments.

Finally we would like to mention that, although we have focussed on the force experienced by the probe, the studies [6] performed to explain the Au NP displacements calculated the force on the target, using the Maxwell stress tensor formalism. We have compared both forces and for semi-infinite targets we have found that for metals they are equal, 
i.e., they are action-reaction forces. Nevertheless, for the case of $\mathrm{MgO}$ where Cherenkov radiation is produced, the transverse force on the target is slightly larger than the force on the probe, so that the momentum conservation law is fulfilled when the momentum carried by $\mathrm{CR}$ is considered. In the case of films, we have proved that both forces are equivalent also for $\mathrm{MgO}$.

\section{Conclusions}

In this work the retardation effects on the interaction between fast electrons and planar films have been explored. First the longitudinal force and its spectral form reflected in the EEL spectra have been studied for metals and insulators sustaining CR. Then the transverse component has been calculated, finding that it is always attractive. Nevertheless, we have also proved the relevance of including the contribution of the magnetic field and of using Kramers-Kronig consistent dielectric functions, warning about the need for checking the later point when using tabulated data, specially for small impact parameters. For point probes the force is found to be attractive in all the cases, but for very extended beams the interaction turns out to be repulsive at small impact parameters, even at moderate relativistic velocities, in agreement with recent studies, but these conditions are far from the setup commonly used in STEM.

\section{Acknowledgements}

This project was supported by the ETORTEK project NANOIKER from the Basque Government (BG), project FIS2010-19609-C02-01 from the Spanish Ministry of Science and Innovation and grant IT-75613 from BG-UPV/EHU. Computational resources were provided by DIPC (UPV/EHU, MICINN, BG, ESF). 


\section{REFERENCES}

1. J.M. Cowley, Ultramicroscopy 9 (1982) 231.

2. L.D. Marks, Solid State Commun. 43 (1982) 727.

3. P.M. Echenique and A. Howie, Ultramicroscopy 16 (1985) 269.

4. R. García-Molina, A. Gras-Marti, A. Howie and R.H. Ritchie, J. Phys. C 18 (1985) 5335.

5. A. Rivacoba and P.M. Echenique, Ultramicroscopy 26 (1988) 389.

6. A. Reyes-Coronado, R. Barrera, P.E. Batson, P.M. Echenique, A. Rivacoba and J. Aizpurua, Phys. Rev. B 82 (2010) 235429.

7. P.E. Batson, A. Reyes-Coronado, R.G. Barrera, A. Rivacoba, P.M. Echenique and J. Aizpurua, Nano Lett. 11 (2011) 3388.

8. P.E. Batson, A. Reyes-Coronado, R.G. Barrera, A. Rivacoba, P.M. Echenique and J. Aizpurua, Ultramicroscopy 123 (2012) 50

9. F.J. García de Abajo, Phys. Rev. B. 70 (2004) 115422.

10. P. Rebernik Ribič, Phys. Rev. Lett. 109 (2012) 244801.

11. A. Rivacoba and N. Zabala, New Journal of Physics 16 (2014) 73048.

12. P. Moreau, N. Brun, C.A. Walsh, C. Colliex, A. Howie, Phys. Rev. B 56 (1997) 6774.

13. R.H. Ritchie, Phys. Rev. 106 (1957) 874.

14. D.M. Roessler and W.C. Walker, Phys. Rev. 159 (1967) 733.

15. N. Zabala, A.G. Pattantyus-Abraham, A. Rivacoba, F.J. Garcia de Abajo and M.O. Wolf Phys. Rev. B 68 (2003) 245407.

16. E.D. Palik, Handbook of Optical Constants of Solids (Orlando: Academic Press, 1985).

17. W.S.M. Werner, K. Glantschnig and C. Ambrosch-Draxl, J. Phys. Chem. Ref. Data 38 (2009) 1013. 
Figure 1. (a) Energy loss probability per unit length for $300 \mathrm{keV}$ electrons parallel to Al slabs of thicknesses $a=1,10$ and $100 \mathrm{~nm}$ at impact parameter $b=1 \mathrm{~nm}$. The sketch in the inset represents the geometry of the problem. (b) Dispersion relation for $300 \mathrm{keV}$ electron in front of an $\mathrm{Al}$ film of thickness $a=20 \mathrm{~nm}$ (red) and a semi-infinite medium (blue). Dashed lines correspond to non-retarded approximation in both (a) and (b).

Figure 2. Energy loss probability per unit length experienced by $300 \mathrm{keV}$ electrons moving close to $\mathrm{MgO}$ slabs of thickness (a) $a=10$, (b) $a=100 \mathrm{~nm}$ and (c) $a=1 \mathrm{~mm}$ at impact parameter $b=1 \mathrm{~nm}$ (red). The blue lines represent the corresponding calculations for a semi-infinite medium. The sketch in the inset of the figure (b) represents the confinement of Cherenkov radiation in the film. In figure (c) the black line corresponds to $b=10 \mathrm{~nm}$. The dashed lines represent the corresponding non-retarded approximations.

Figure 3. (a) Transverse force experienced by $300 \mathrm{keV}$ electrons moving close to a $\mathrm{MgO}$ film of thickness $a=1 \mathrm{~nm}$ as a function of impact parameter. The $\omega$ component of the force is represented in the inset. (b) The same for Au slabs of $a=1 \mathrm{~nm}$ (black) and $a=10 \mathrm{~nm}$ (red) but using Werner's dielectric function [17]. For $a=1 \mathrm{~nm}$ the same is represented (green line) but using Palik's dielectric function [16]. The dashed lines represent the corresponding non-retarded expressions. 

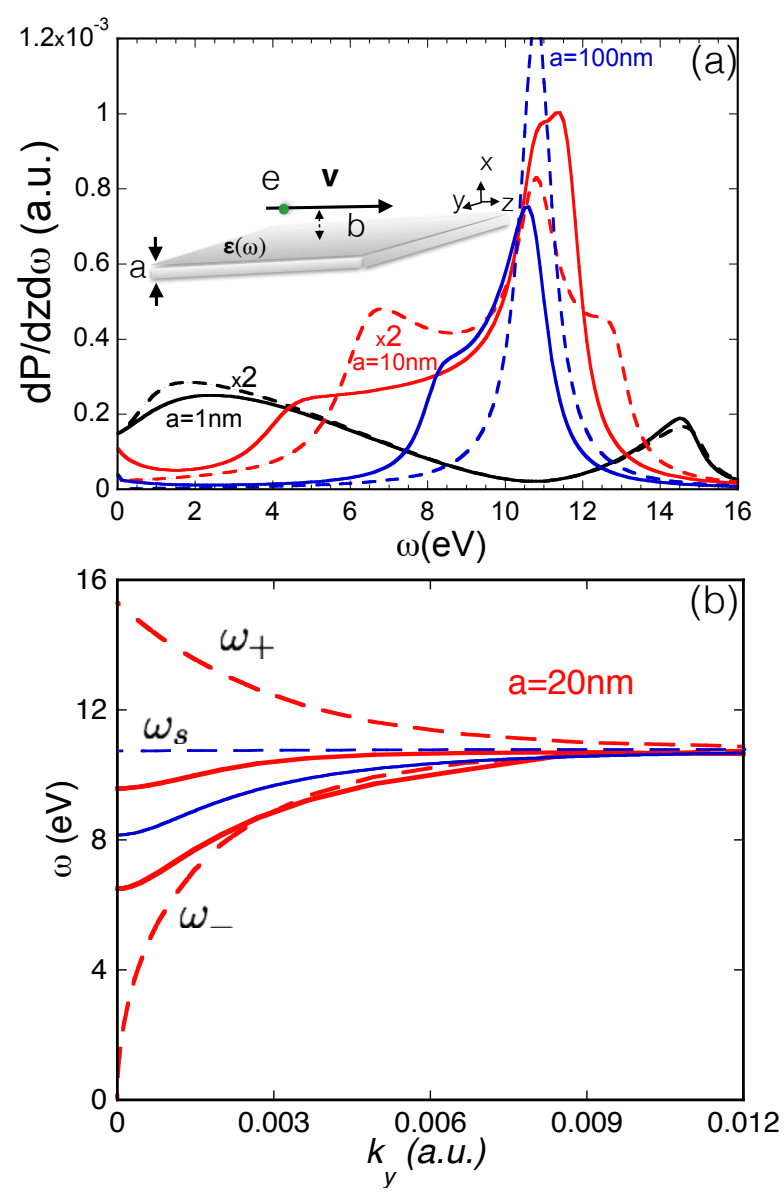

FIGURE 1 


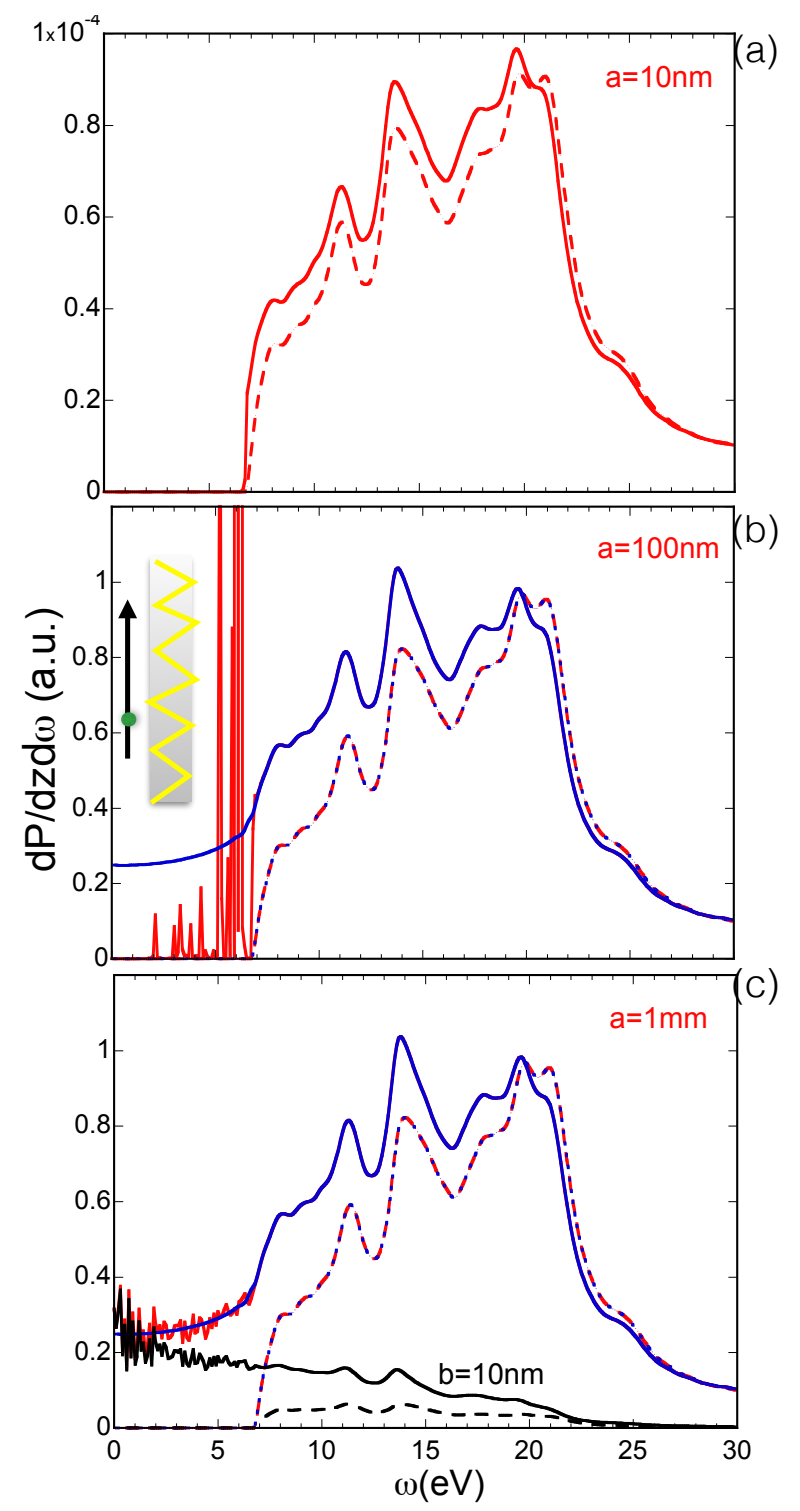

FIGURE 2 


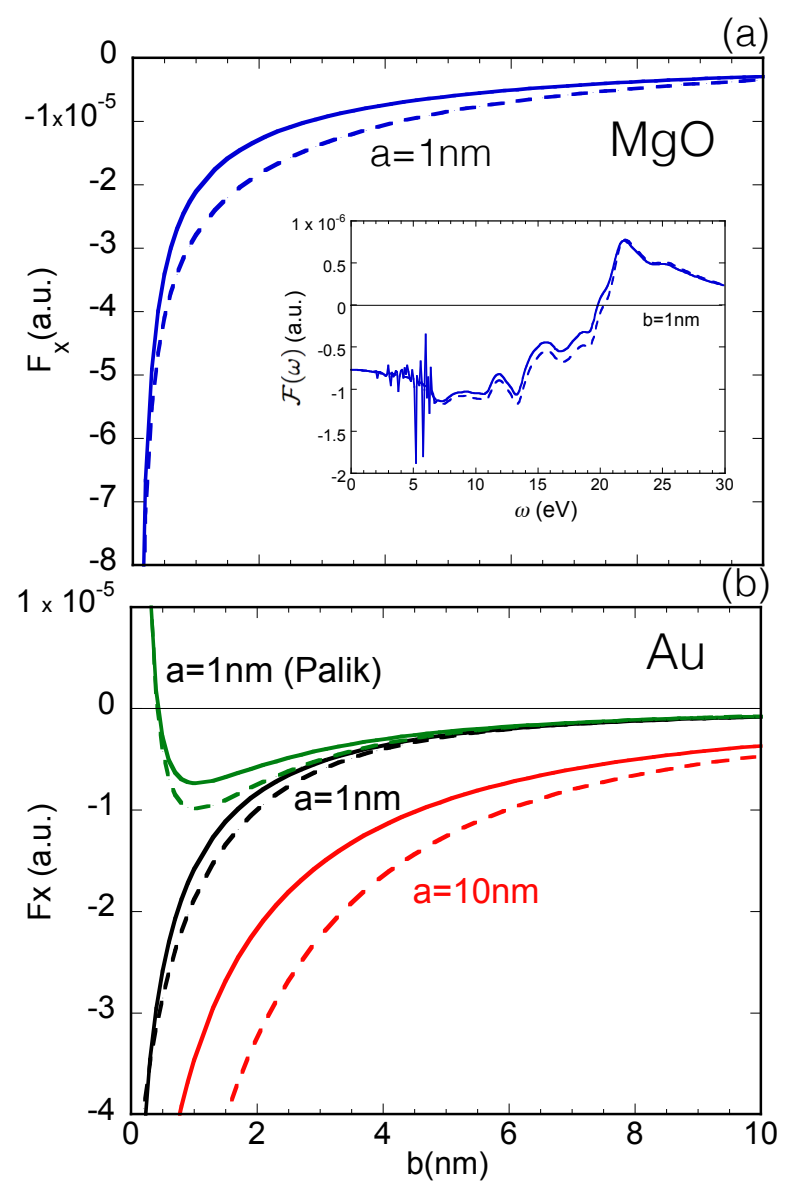

FIGURE 3 